\title{
Diderot et l'Antiquité classique, dir. A. Lehmann
}

\section{Giuseppina D'antuono}

\section{(2) OpenEdition}

\section{Journals}

\section{Edizione digitale}

URL: https://journals.openedition.org/studifrancesi/21296

DOI: 10.4000/studifrancesi.21296

ISSN: 2421-5856

\section{Editore}

Rosenberg \& Sellier

\section{Edizione cartacea}

Data di pubblicazione: 1 décembre 2019

Paginazione: $575-576$

ISSN: 0039-2944

\section{Notizia bibliografica digitale}

Giuseppina D'antuono, «Diderot et l'Antiquité classique, dir. A. Lehmann», Studi Francesi [Online], 189 (LXIII | III) | 2019, online dal 01 mars 2020, consultato il 11 novembre 2021. URL: http://

journals.openedition.org/studifrancesi/21296 ; DOI: https://doi.org/10.4000/studifrancesi.21296

Questo documento è stato generato automaticamente il 11 novembre 2021.

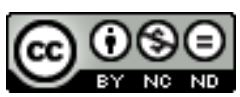

Studi Francesi è distribuita con Licenza Creative Commons Attribuzione - Non commerciale - Non opere derivate 4.0 Internazionale. 


\title{
Diderot et l'Antiquité classique, dir. A. Lehmann
}

\author{
Giuseppina D'antuono
}

\section{NOTIZIA}

Diderot et l'Antiquité classique, dir. A. Lehmann, Paris, Classiques Garnier, 2018, 388 pp.

L'appello ai posteri «Non omnis moriar; multaque pars mei vitabit Libitinam» che Diderot condivise con Orazio, affinché il suo nome restasse e non morisse tout entier (Orazio, Odi, III, 30, vv. 6-7) è ben noto agli studiosi del philosophe. Orbene quel desiderio di matrice classica di colloquiare con gli antichi e con i posteri è al centro del denso volume Diderot et l'Antiquité classique, curato da Aude Lehmann, e apparso nella collana Rencontres, per la serie Dix-huitième siècle, che, diretta da J. Berchtold e C. Seth, conta già una trentina di titoli. I 17 contributi presentati, nelle giornate del 4 e 5 ottobre 2013 a Mulhouse, nel Colloquio omonimo, inteso a portare avanti le riflessioni e le ricerche dei latinisti e dei grecisti su Diderot e rendergli omaggio nell'anno delle celebrazioni del tricentenario della nascita, appaiono distribuiti in quattro sezioni.

Nell'Avant-Propos la curatrice del volume, A. Lehmann, dopo aver illustrato le ragioni del convegno, mette a fuoco, come oggetto di riflessione e di dibattito futuro, le idee che tornano nel suo contributo e che dalle prime battute sostengono l'impianto del volume. L'idea principale è che occorresse riesaminare l'opera del Langrois dalla prospettiva degli antichisti, beninteso non degli eruditi né degli anticomani (termine più volte criticato), quanto degli esperti di antichità versati in storia antica e filologia. Durante tutto il secolo $\mathrm{xx}$, in particolare a partire dagli anni Trenta, e fino alla fine degli anni Novanta, dei profondi solchi in tale prospettiva sono certo stati tracciati da studiosi del calibro di Thomas, Seznec, Marchal, Baudiffer, Dieckmann, Trousson i quali hanno messo in luce l'importanza della famiglia di origine di Diderot, del padre fiero coltellinaio, il percorso di studi compiuto presso i Gesuiti e la funzione mediatrice della lingua latina, nonché gli autori antichi preferiti. Occorreva tuttavia andare oltre tali 
acquisizioni e interrogarsi sulla funzione delle antichità nell'elaborazione di una specifica visione del mondo, di una particolare moralità e di un'idea estetica, politica e filosofica del nostro. Era insomma necessario chiedersi se e come gli autori antichi avessero orientato una certa formazione del philosophe. A nessuno degli autori dei contributi, Diderot appare come un ammiratore beato né, tantomeno, un cieco adulatore degli antichi; a suffragare questa impressione, osserva la Lehmann, emerge nelle opere diderotiane una costante tensione dialettica tra antico e moderno, tra passato e presente che orienta una funzionalità politica del testo.

Nel primo contributo di Robert Bedon Diderot et l'article Langres de l'Encyclopédie (pp. 19-46), l'interesse è rivolto alla civiltà gallo-romana del paese natale e alla forma che essa assunse nel discorso diderotiano. Bedon enuclea i motivi che lo hanno condotto ad attribuire la paternità dell'articolo a Diderot, esponendo le vestigia, simbolo delle Antichità di Langres, e scegliendo di avvicinarle alla storia tragica di due amanti originari del luogo. Le fonti usate per l'articolo e soprattutto la denuncia delle vestigia sottratte, allorché Colbert fu ministro, sono il segno dell'interesse nutrito, a parere di Bedon, dal Nostro per la sua terra natale. Jean-Marie André in Diderot et l'Antiquité romaine. Antiquités et Antiquité (pp. 49-67) spiega piuttosto un certo bisogno di storicità presente in Diderot, insistendo sul fatto che occorre distinguere tra le Antichità e non le antichità, per sottolineare come non si tratti di un culto superficiale, perché l'arte antica non conduce a un culto mondano, erudito, ma alla scienza e a una resurrezione parziale del passato. Ciò che conta è il tentativo di tracciare un simbolo della memoria. La storia insomma serve a costruire un sogno, costituito da Roma, per contrastare la tirannia e diffondere le idee di libertà. L'influenza dell'estetica di Aristotele su Diderot è l'oggetto di analisi del contributo di M.A. Zagdoun (Diderot et l'esthétique d'Aristote, pp. 75-91), che specifica come tale influenza sia limitata al rispetto di talune regole del classicismo, che impongono naturalezza e semplicità sulla scena. Non vi è accordo invece nell'estetica dei caratteri, poiché in tal senso la riflessione drammaturgica diverge. Eppure sarebbe errato definire Diderot un classicista. Infatti, meritoriamente la Zagdoun richiama l'attenzione del lettore sui diversi contesti storici di Aristotele e Diderot, che restano l'elemento distintivo nella formazione di diverse idee estetiche.

5 L'estetica diderotiana è al centro anche dell'articolo di Dominique Bocage-Lefèbvre ( $L a$ célébration de l'Antiquité dans trois cuvres esthétiques de Diderot: "Essais sur la peinture", "Éloge de Térence", "Pensées détachées sur la peinture", pp. 93-107) che ha posto sotto la sua lente analitica tre opere, per misurarne in chiave comparativa l'ammirazione per le Antichità, che non è mai espressa in modo rigoroso. Terenzio, come Fedro, Epitteto, caduti in schiavitù perché difensori della patria, costituiscono riferimenti intertestuali costanti e hanno un ruolo di rilievo come simboli nella scrittura diderotiana alla ricerca della verità. Il terzo contributo dedicato all'estetica del Langrois è quello di Nadège Neumuller (Diderot et l'histoire plinienne des arts, pp. 109-133) che ha focalizzato l'influsso della Storia di Plinio nella formazione di un ideale molto lontano dall'Horresco referens virgiliano. È parere dell'autore che la formula di Seznec, che distingue il piano poetico da quello artistico, sia lo specchio di quanto Diderot credesse, in linea con Plinio. Un'opera, per definirsi bella, non doveva solo rispettare le regole ed essere fedele al modello da rappresentare, ma anche aveva il dovere di veicolare emozioni e pathos. L'interesse per Plinio coinvolge anche Assembé Ela, autore del contributo Un paragon oublié, Diderot-Falconet-Pline (pp. 135-160). La voce materiale, fonte concreta, è il collante 
che tiene insieme i tre protagonisti dell'articolo. Sono interrogate le fonti letterarie e poetiche comuni, ma anche quelle diverse, per dimostrare quanto la critica sia essenzialmente un'arte della parola, che racconta l'immagine e che non sia più mera retorica come in Falconet. L'ekphrasis diventa così, per il Nostro, un'arte scientifica, un vero genere letterario.

6 La parola, intesa come azione nel dialogo, è l'oggetto del saggio Le dialogue diderotien avec Aristote et Platon (pp. 163-175) che inaugura la seconda parte del volume. L'autrice Sophia Felopoulou mostra come il Langrois in forza più delle teorie platoniche che di quelle aristoteliche, sia riuscito a rinnovare il teatro e a tracciare il cammino del dramma contemporaneo tanto nella teoria che nella pratica della scena. Ben due contributi sono riservati al complicato rapporto Seneca-Diderot. Il primo di Christine Hammann (Sénèque avocat de Diderot, procureur de Rousseau, pp.177-188) analizza il rapporto che Diderot e Rousseau ebbero con Seneca, entrambi molto presi dalla persona e dall'opera dello stoico latino, eppure così distanti nei loto giudizi al riguardo. Diderot, che si era avvicinato a Seneca fin dal 1747, cambiò la prospettiva da cui guardare allo scrittore romano dopo il ritorno dalla Russia. Lettore della vita del precettore di Nerone, ne comprese le ragioni; Rousseau, che lesse le opere di Seneca, in un'ottica moralizzatrice, ne stigmatizzò in toto l'operato. Un Seneca oggetto di discussione nel salotto di d'Holbach è messo a fuoco nelle pagine di Cecile Merckel ( $\mathrm{L} a$ figure de Sénèque dans l"'Essai sur les règnes de Claude et de Néron" de Diderot, pp. 189-204), come incoerente, espressione di una personalità complessa e contraddittoria. Tuttavia Diderot nel suo Essai sur les règnes de Claude et de Néron vuole mostrare l'identità e l'unità del carattere del filosofo latino e così studiando le sue opere, in particolare le epistole, mostra quanto fosse un uomo virtuoso. Proseguendo su questa linea di lettura, la ricerca della verità sembra a noi essere l'interessante contributo di Valérie Perez (Le jeu agonistique de la parole. Diderot et le parler-vrai, pp. 205-220), che disamina doviziosamente la funzione della parresia nella sua fase evolutiva, ovvero, nei colloqui tra Diderot e Caterina II. Il parler-vrai che conduce il Nostro alla individuazione del vero Seneca è un tratto caratteristico del suo discorso politico e drammaturgico.

7 Davvero un bel cammeo è il saggio L'inspiration pindarique ou la composition personnifiée di Tatiana Smoliarova (pp. 223-241) che getta una luce nuova sull'uso dell'ode anacreontica e del verso pindarico in Les Éleuthéromanes di Diderot, recitato nel salotto di Holbach nel 1772. Il componimento di chiara ispirazione tirannicida alla Meslier ebbe una discreta fortuna in epoca rivoluzionaria direttoriale, per essere obliato e recuperato a fine XIX secolo e qui ci corre l'obbligo di segnalare un'ulteriore edizione del testo del 1884 non menzionata dall'autrice. La poesia torna alla sua arcaicità tenendo insieme danza, musica, gesto e declamazione. La poesia è viatico fondamentale per il teatro e Sofocle e Filotette sono funzionali alla costruzione di un nuovo teatro, così come mostra Marie Saint Martin ( «La vérité! La nature! Les Anciens! Sophocle! Philoctète!» Diderot et l'Antiquité, l'invention d'une modernité à l'antique, pp. 243-260). A tal fine anche lo studio di Terenzio fu fondamentale per riformare il teatro e questo è l'oggetto del saggio di Gualtiero Calboli (L'Essai "Sur Térence" de Diderot et la "Vie de Térence" chez Donat. Étude critique et comparée, pp. 261-280). La conoscenza diderotiana, come emerge, era molto approfondita degli scritti terenziani, egli amava molto l'autore di Heautontimorumenos, perché vi trovava le sue convinzioni estetiche ed etiche. Il metodo di Diderot in rapporto agli antichi non rasentava mai l'imitazione. Come spiega Marilina Gianico ("Avons-nous plus de délicatesse et plus de génie que les Athéniens?» L'Antiquité classique, légitimation de la réforme théâtrale diderotienne, pp. 281-298) si 
trattava di elaborare una poetica della rappresentazione patetica. Sebbene il philosophe non prediligesse i generi teatrali classici e lavorasse per la nascita del nuovo dramma, a parere di Houda Landolsi (Suzanne Simonin, une héroïne de l'Antiquité?, pp. 301-316), egli fu autore di una sorta di tragedia che appare nella Religieuse, in cui Suzanne Simonin, la protagonista, è l'eroina di una nuova forma di teatro alla greca. Alla forte presenza di un Cicerone, politico sacrificatosi per la patria, si dedica Lehmann (L'image de Cicéron dans l'œuvre de Diderot, pp. 317-346), mentre alla figura di Celso nell'Encyclopédie è dedicato il contributo di Aurélian Gautherie (Celse et le "De Medicina" dans l"'Encyclopédie" de Diderot, pp. 347-362). Un valore aggiunto vogliamo riservare ai sei funzionali indici, con cui si chiude il volume. Questa miscellanea ha, ci sembra, colto quell'obiettivo eternizzante, che è sempre sotteso alla scrittura diderotiana, che il Langrois aveva ereditato degli Antichi, e che appare in maniera particolarmente in questo brano di una lettera del 1766 «Ce qui échappe à nos contemporains n'échappera pas à l'œil du temps et de la posterité». Grazie anche alle ricerche raccolte in questo volume, la posterità è oggi più consapevole dell'apporto etico, politico e sociale degli Antichi, attraverso le cui scritture Diderot intese costruire, come confidava a Galiani, «un projet pour ramener ses concitoyens dissolus aux vertus de leurs premiers ancêtres». 